\title{
A catalogue of calibrator stars for long baseline stellar interferometry ${ }^{\star}$
}

\author{
P. Bordé ${ }^{1}$, V. Coudé du Foresto ${ }^{1}$, G. Chagnon ${ }^{1}$, and G. Perrin ${ }^{1}$ \\ LESIA, FRE2461, Observatoire de Paris, 5 place Jules Janssen, 92195 Meudon, France \\ e-mail: Vincent.Foresto@obspm.fr; Gilles.Chagnon@obspm.fr; Guy.Perrin@obspm.fr
}

Received 12 March 2002 / Accepted 27 June 2002

\begin{abstract}
Long baseline stellar interferometry shares with other techniques the need for calibrator stars in order to correct for instrumental and atmospheric effects. We present a catalogue of 374 stars carefully selected to be used for that purpose in the near infrared. Owing to several convergent criteria with the work of Cohen et al. (1999), this catalogue is in essence a subset of their self-consistent all-sky network of spectro-photometric calibrator stars. For every star, we provide the angular limb-darkened diameter, uniform disc angular diameters in the $J, H$ and $K$ bands, the Johnson photometry and other useful parameters. Most stars are type III giants with spectral types $K$ or M0, magnitudes $V=3-7$ and $K=0-3$. Their angular limb-darkened diameters range from 1 to 3 mas with a median uncertainty as low as $1.2 \%$. The median distance from a given point on the sky to the closest reference is $5.2^{\circ}$, whereas this distance never exceeds $16.4^{\circ}$ for any celestial location.
\end{abstract}

Key words. catalogs - stars: fundamental parameters - instrumentation: interferometers - techniques: interferometric

\section{Interferometry and calibration}

A stellar interferometer collects and recombines light from separate apertures. The interferometric observable, called the visibility, is a measure of the spatio-temporal coherence of the target. It is a complex quantity whose modulus and phase are respectively derived from the contrast and the position of the fringe packet resulting from the recombination process. In order to take into account instrumental and atmospheric effects, observations of the target should be bracketed by observations of calibrator stars. Thus, the object visibility $V_{\star}$ can be deduced from the measured object and reference fringe contrasts $\mu_{\star}$ and $\mu_{\text {ref by }}$

$V_{\star}=\frac{\mu_{\star}}{\mu_{\text {ref }}} V_{\text {ref }}$,

provided the reference visibility $V_{\text {ref }}$ can be predicted at the time of the observations. This in turn implies two things:

1. A model for the reference source has been chosen.

2. The uncertainties on the model parameters are desired to be small enough not to dominate the measurement error budget.

Send offprint requests to: $\mathrm{P}$. Bordé, e-mail: Pascal.Borde@obspm.fr

* The catalogue is only available in electronic form at the CDS via anonymous ftp to cdsarc.u-strasbg.fr $(130.79 .128 .5)$ or via http://cdsweb.u-strasbg.fr/cgi-bin/qcat?J/A+A/393/183
A single star appears as a straightforward reference source whose simplest model is a uniform disc (UD) fully described by its angular diameter (single-parameter model). In the following, we will call calibrator star such a reference source. In this case, the visibility $V_{\text {ref }}$ is given by

$V_{\text {ref }}=\frac{2 J_{1}(x)}{x}$ with $x=\frac{\pi B \theta_{\mathrm{UD}}}{\lambda}$,

where $B$ is the interferometric baseline projected on the sky, $\theta_{\mathrm{UD}}$ the stellar angular diameter and $\lambda$ the wavelength. A more elaborate model would be a limb-darkened disc (LDD) with two parameters, the stellar angular diameter and either the coefficient of a linear law or the exponent of a power law for limbdarkening (e.g. Hestroffer 1997). However, for the purpose of calibration, the UD model is in general fully satisfactory provided the visibility is large enough. For instance, if $V \geq 50 \%$, UD and LD models do not differ by more than $1 \%$ in the $K$ band (Perrin et al. 1998).

To improve calibration, $\mu_{\star}$ and $\mu_{\text {ref }}$ should be measured with the same instrumental and preferably atmospheric conditions, hence the need for a catalogue of calibrator stars with a sky coverage as complete as possible. Therefore, in the framework of the simplest single-parameter UD model, a dense grid of reference stars along with their angular diameters has to be worked out. Since only few stellar angular diameters have been directly measured so far at a precision better than $5 \%$, it seems unavoidable to rely also on indirect measurements. Two approaches are possible: collecting all existing observations and compiling them (e.g. the CHARM catalogue by 
Richichi \& Percheron 2002), or selecting carefully a few hundred stars with well known characteristics and deduced diameters. The first approach leads to a larger but more heterogeneous catalogue with one entry per observation, whereas in the second one, one star corresponds to one entry and all diameters are given with comparable precision. The last option was chosen for the work presented in this paper. It originated in the need of a grid of calibrator stars among the FLUOR interferometry team. As this need arises for every interferometer, we thought of making this grid available to other interferometry teams through publication. FLUOR, the Fiber Linked Unit for Optical Recombination, is currently hosted at the IOTA, the Infrared and Optical Telescopes Array (Traub 2000), and is described by Coudé du Foresto et al. (1998). The IOTA features baselines between 7 and $38 \mathrm{~m}$ and FLUOR operates in the $K$ and $L$ bands.

In the next section, we describe the requirements that any star would ideally fulfill to be selected as a calibrator. In Sect. 3, we briefly review the work done by Cohen et al. (1999) (tenth paper of a series, hereafter referred to as C.X) and we detail the way we extracted a sublist of calibrator stars. Section 4 deals with statistical characteristics of the stellar sample in terms of sky coverage or magnitude and angular diameter distributions. Section 5 is dedicated to the catalogue format, the calculation of the UD diameters and the definition of some specific quantities. Finally, we compare angular diameter determinations by C.X with existing measurements in Sect. 6.

\section{Selection criteria for reference sources}

We have argued that any source with a predictable visibility can be chosen as a reference. In that sense, a double star can be a good candidate provided all orbital parameters are known with enough accuracy. However for reliability as well as practical reasons, we prefer to reject everything but single non-variable stars with compact atmospheres. Indeed, variable stars are either multiple or change diameters with time, and extended atmospheres would lead to non trivial visibility models. In those cases, one may risk that the formal error bars would not represent the true errors because of the inadequacy of the chosen model. All this can be expressed in terms of the following observational constraints that remain to be quantitatively precised:

- no evidence of multiplicity;

- radial velocity stability;

- photometric stability;

- no evidence of infrared excess;

- evidence of a compact atmosphere;

- accurately determined angular diameter.

These requirements become relaxed as the source gets smaller, the baseline shorter and the wavelength longer. As we shall see, angular diameters can be computed thanks to spectrophotometric models.

\section{From spectro-photometric to interferometric references}

For their all-sky radiometric network, C.X have defined a set of criteria very close to those of Sect. 2. Moreover, they have derived angular diameters for all their stars, and found an excellent agreement between their predicted diameters and those measured by interferometry. It seems very attractive to use their network as the core of a catalogue of calibrators meant for interferometry. With this goal in mind, let us review briefly some key points of their work.

C.X have assembled composite spectra for a dozen giant stars from observed fragments in various spectral bands. These spectra are absolutely calibrated with respect to their primary IR standard, Sirius, and thus constitute a set of secondary standards. Fitting Kurucz model atmosphere (Kurucz 1993) to these spectra yields angular diameters with formal uncertainties. Now, C.X make the fundamental hypothesis that the deredenned spectrum of a giant star with a given spectral type (within the range K0-M0) can serve as a template for every other star with the same spectral type (the so-called "template assumption"). Then, infrared photometric measurements in several bands provide the correct scaling factor for the corresponding template. With the goal of finding at least one candidate per $50 \mathrm{deg}^{2}$ on the sky, they have searched the IRAS database with the following criteria:

- a minimal infrared luminosity of $1 \mathrm{Jy}$ at $25 \mu \mathrm{m}$;

- a position in the infrared color-color diagram defined as "normal" by Walker \& Cohen (1988);

- declared as non-variable (with a probability over 90\%), not an emission-line object or carbon star;

- isolated object (other sources within $6^{\prime}$ should not contribute to more than $5 \%$ of the total flux at 12 and $25 \mu \mathrm{m}$ );

- away from a field of extended infrared emission;

- restriction on spectral type (K0-M0) and luminosity class (II-IV).

For all the 422 selected sources (referred to as Cohen's list in the following) they have collected accurately calibrated infrared photometric measurements, and scaled every synthetic spectrum. Angular diameters are easily derived as the square root of the scaling factor times the template's diameter.

In addition to previously mentioned spectro-photometric criteria, the interferometric context requires that a single star would be fed into the collecting apertures. Therefore, acceptable stars could be part of a multiple system provided the separations between the components are wider than the interferometric field of view, or provided the interferometer would not be able to resolve the system. For reliability reasons, we shall not allow binary systems of the last category in this work. Since the interferometric field of view of single-mode interferometers, like FLUOR, is of the order of an Airy disc, angular separations of a few $\lambda / D$ could theoretically be suitable. However, the pointing accuracy of the telescope, the ability of the tracking system to remain locked on the right component and the confusion risk between close stars should also be taken into account. Therefore, considering a pointing accuracy of $\approx 1^{\prime \prime}$ and average seeing conditions $\left(1-1.5^{\prime \prime}\right)$, we have chosen to discard 
all double stars with separations less than $4^{\prime \prime}$, regardless of the difference in magnitude between the components. Concerning double stars with both components in the field of view of the tracking system, $30^{\prime \prime}$ in the case of IOTA, we shall require a difference in visual magnitude of 5 between the components (i.e. the secondary contribute to $1 \%$ or less to the total visual flux).

We have investigated the nature of all the sources in Cohen's list with the Simbad database and found that all stars belong to one of the twelve types ordered in Table 1. When a new source which could be classified under several object types is added to the Simbad database, a unique choice is made according to the hierarchical classification proposed by Ochsenbein \& Dubois (1992). Table 1 states the priority order for our twelve object types. For instance, a variable star in a double system would be classified as variable, and a spectroscopic binary in a cluster would be classified as a spectroscopic binary.

Stars belonging to categories 1 to $5(14.09 .05 .4,14.09 .04 .5$, 12.13.01.2, 14.09.05.0, 14.09.01.0) were removed because of their identified variability. All 12 spectroscopic binaries were double-checked with the Batten catalogue (1989) and also removed. Since all stars that are stated as Variable (14.09.00.0) by Simbad were investigated by Cohen and declared as non variable (with a probability over 90\%), their supposed variability is likely to be due to instrumental uncertainties, so those stars were not discarded for this reason only. However, because of Simbad classification rules, a Variable star or a High proper motion star may also be in a double or multiple system. That is why all stars in Cohen's list have been searched for in the Catalogue of Visual Double Stars in Hipparcos (Dommanget \& Nys 2000). We have then applied the selection criteria previously established to all binary systems found. This procedure led us to reject all Double or multiple stars (12.13.00.0), 4 stars in double system (14.04.00.0), 3 variable stars (14.09.00.0) and 3 stars (14.00.00.0). For all stars in double system (14.04.00.0) and stars of other categories that revealed themselves to be multiple with components within 1', our catalogue provides the separation and the difference in visual magnitude of the companions (in the field COMTS, see Table 5). The 3 stars in a cluster (14.01.00.0) have been kept because each of them happen to be alone in a $1^{\prime}$ field. As a result, our interferometric catalogue is left with 374 entries.

\section{Stellar sample properties}

\subsection{Statistical data}

Let us now discuss some general properties of our catalogue. Figure 1 displays its sky coverage. The maximum distance from a point on the sky to the closest calibrator star is $16.4^{\circ}$, whereas the median distance is $5.2^{\circ}$. Most stars (91\%) are class III giants (see histogram in Fig. 2) and all have a spectral type $K(82 \%)$ or M0 (18\%) (see histogram in Fig. 3). The majority of stars $(72 \%)$ have a visual magnitude between 4 and 6 , and almost all of them (95\%) between 3 and 7, with a median value of 5.0 (see histogram in Fig. 4). As for $K$ magnitude, most stars $(95 \%)$ lie in the interval $K=0-3$ with a median

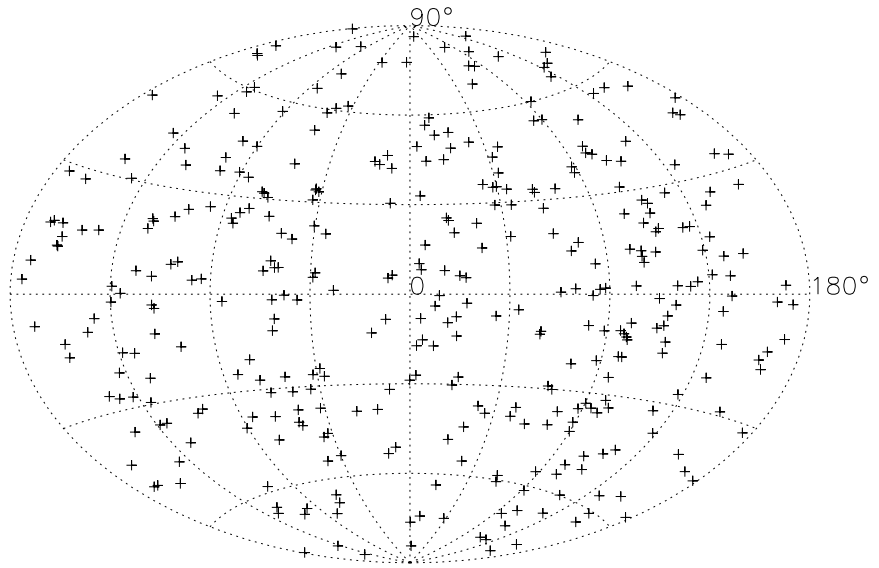

Fig. 1. Sky coverage of reference stars in the catalogue (HammerAitoff equal-area projection).

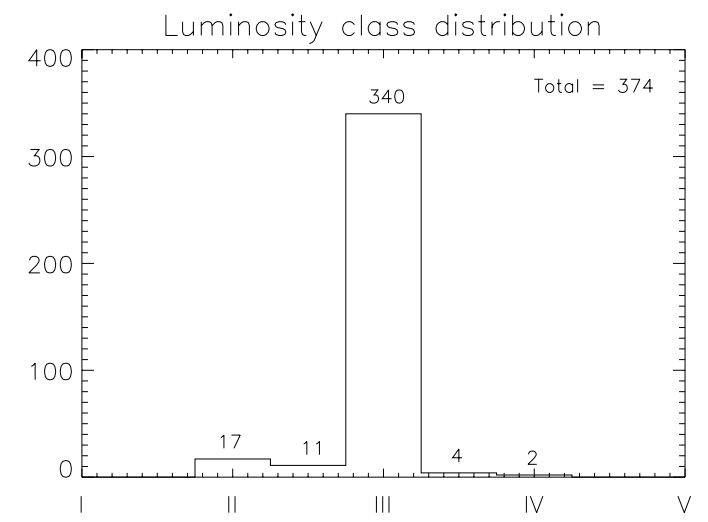

Fig. 2. Distribution of stars in the catalogue as a function of their luminosity class. Almost all stars (91\%) are class III giants.

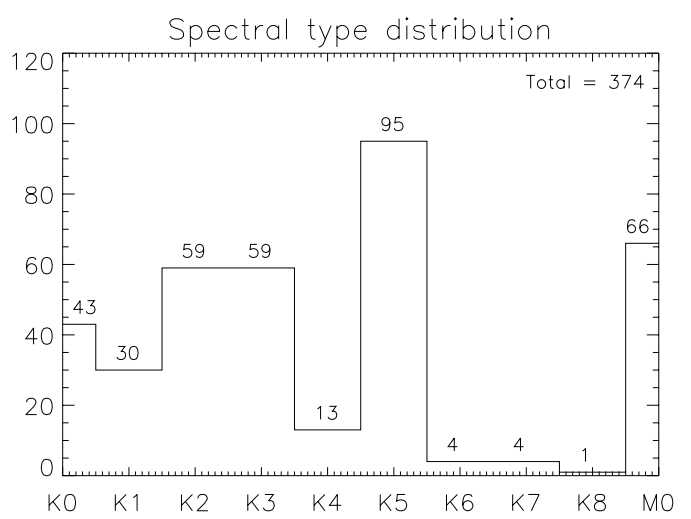

Fig. 3. Distribution of stars in the catalogue as a function of their spectral type. The sample is dominated by K2-K3 (32\%), K5 (25\%) and M0 (18\%).

value of 1.8 (see histogram in Fig. 5). Limb-darkened angular diameters (see Sect. 5.2 below) range from 1 to 10 mas with a median value of 2.3 mas (see histogram in Fig. 6). The median uncertainty on the diameter is $1.2 \%$ (see histogram in Fig. 7) for LD and UD diameters. 
Table 1. Distribution of stars belonging to the all-sky network by Cohen et al. (1999) as a function of the Simbad object types.

\begin{tabular}{llll}
\hline \hline Priority & $\begin{array}{l}\text { Numeric } \\
(\text { Simbad })\end{array}$ & Standard name & $\begin{array}{l}\text { Nb. of records } \\
\text { (Total = 422) }\end{array}$ \\
\hline 1 & 14.09 .05 .4 & Semi-regular pulsating star & 5 \\
2 & 14.09 .04 .5 & Variable of RS CVn type & 3 \\
3 & 12.13 .01 .2 & Eclipsing binary of Beta Lyrae type & 1 \\
4 & 14.09 .05 .0 & Pulsating variable star & 2 \\
5 & 14.09 .01 .0 & Variable of irregular type & 3 \\
6 & 12.13 .02 .0 & Spectroscopic binaries & 12 \\
7 & 14.09 .00 .0 & Variable star & 99 \\
8 & 14.07 .00 .0 & High proper-motion star & 21 \\
9 & 14.04 .00 .0 & Star in double system & 56 \\
10 & 14.01 .00 .0 & Star in a cluster & 3 \\
11 & 12.13 .00 .0 & Double or multiple star & 12 \\
12 & 14.00 .00 .0 & Star & 205 \\
\hline
\end{tabular}

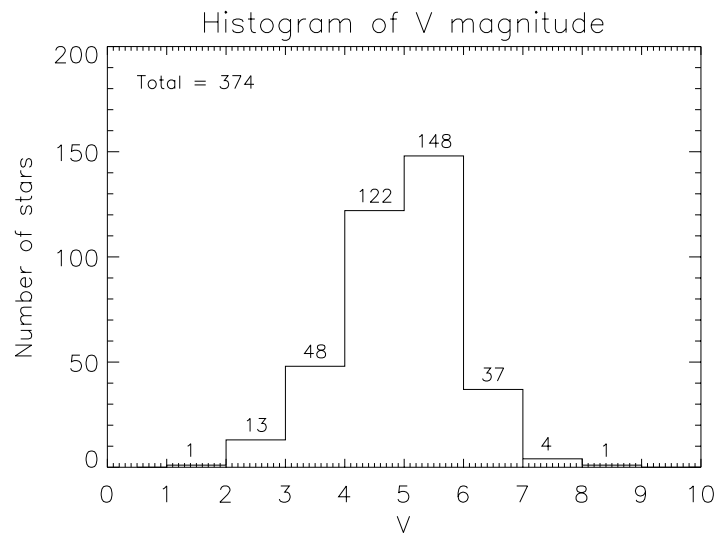

Fig. 4. Distribution of stars in the catalogue as a function of their $V$ magnitude.

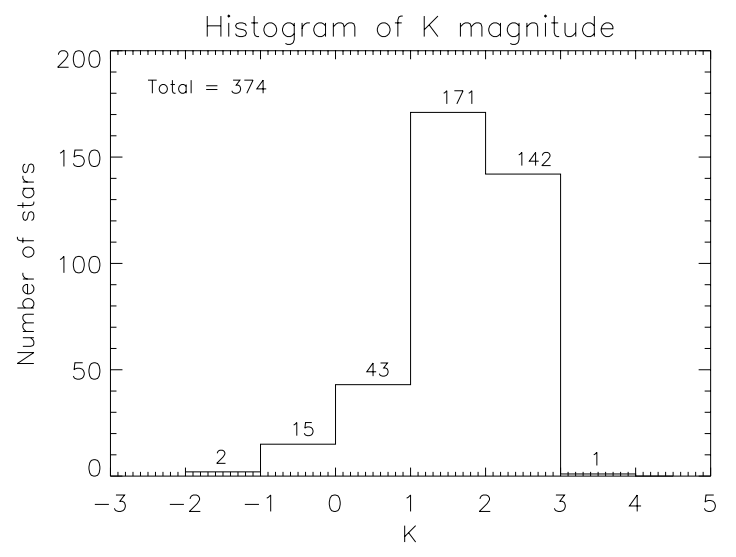

Fig. 5. Distribution of stars in the catalogue as a function of their $K$ magnitude.

\subsection{Suitable interferometric baselines}

The key issue is the maximum interferometric baseline on which a star of our catalogue can be used as a calibrator. Let us take the example of a typical star from our catalogue. If modeled as a uniform disc (UD) in the $K$ band, it would have an angular diameter $\theta_{\mathrm{UD}}=2.27$ mas known with a typical accuracy of $1.2 \%$ (median values). The expected visibility for such

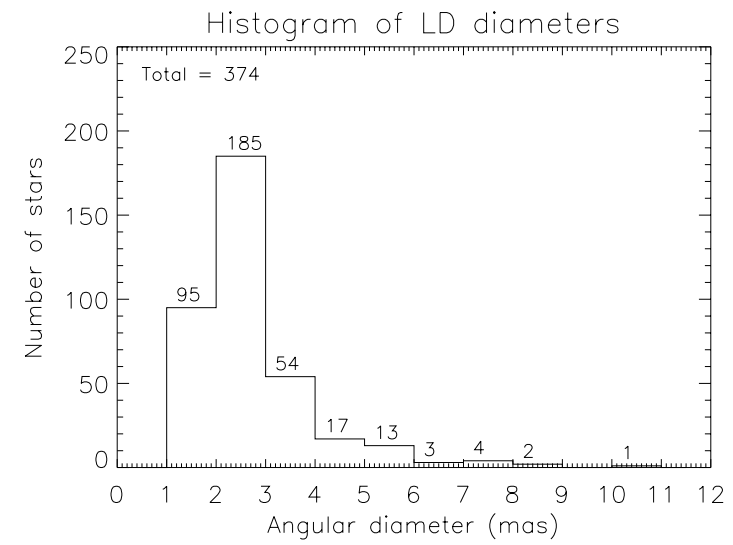

Fig. 6. Distribution of stars in the catalogue as a function of their limbdarkened diameter in milli-arcsec.

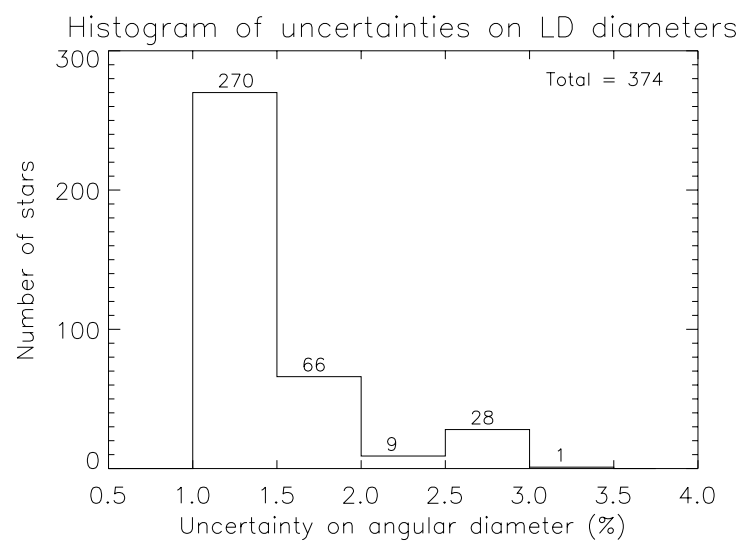

Fig. 7. Distribution of stars in the catalogue as a function of the uncertainty (in percentage) on their limb-darkened diameter.

a diameter is given by Eq. (2) and plotted in Fig. 8. The effect of the uncertainty on the diameter of the calibrator star on the uncertainty on the visibility can be mathematically derived:

$\frac{\Delta V}{V}=x \frac{J_{2}(x)}{J_{1}(x)} \frac{\Delta \theta_{\mathrm{UD}}}{\theta_{\mathrm{UD}}} \quad$ with $\quad x=\frac{\pi B \theta_{\mathrm{UD}}}{\lambda}$ 


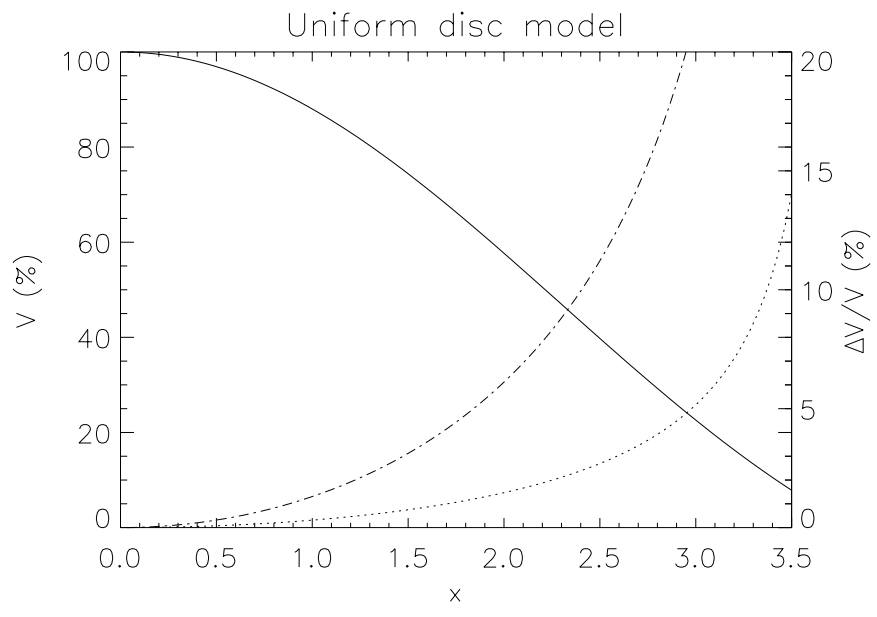

Fig. 8. Visibility curve for a uniform disc model as a function of the reduced variable $x=\pi B \theta_{\mathrm{UD}} / \lambda$ (solid line). Superimposed are the relative uncertainty curves on the visibility, assuming the whole uncertainty is due to the uncertainty on the angular diameter. Two cases are drawn: $\Delta \theta_{\mathrm{UD}} / \theta_{\mathrm{UD}}=1.2 \%$ (dotted line) and $5 \%$ (dash-dotted line).

We have plotted $\Delta V / V$ vs. the baseline in the $K$ band for our typical calibrator star with two values of the uncertainty on its angular diameter (Fig. 8):

1. the catalogue median value of $1.2 \%$;

2. a common value of $5 \%$ (e.g. Perrin et al. 1998 or Boden et al. 2000).

Numerical values for $\Delta V / V$ can be found in Table 2 in three cases: a $35 \mathrm{~m}$-IOTA range baseline, a $100 \mathrm{~m}$-PTI range baseline (see Colavita at al. 1999 for a description of the PTI) and a $200 \mathrm{~m}$-VLTI range baseline (see Glindenmann et al. 2000 for more details on the VLTI). To put this into the perspective of the accuracy of modern interferometers (including statistical and calibration errors), FLUOR routinely achieves visibility measurements with errors of $1 \%$ or less, and for instance PTI recently published error bars of about $3.5 \%$ in average (Boden et al. 2000).

Table 2. Relative uncertainties (in \%) on the visibility solely due to the uncertainty on the angular diameter for several baselines $\left(\theta_{\mathrm{UD}}=\right.$ 2.27 mas, $K$ band).

\begin{tabular}{ccccc}
\hline \hline & \multicolumn{4}{c}{ Baseline (m) } \\
$\Delta \theta_{\mathrm{UD}} / \theta_{\mathrm{UD}}$ & 35 & 100 & 150 & 200 \\
\hline $1.2 \%$ & $0.1 \%$ & $0.8 \%$ & $2.3 \%$ & $6.4 \%$ \\
$5 \%$ & $0.4 \%$ & $3.5 \%$ & $9.4 \%$ & $27 \%$ \\
\hline
\end{tabular}

It should be pointed out that the visibility drops to zero for $x_{0}=3.83$ (or $B \simeq 245 \mathrm{~m}$ for $\theta_{\mathrm{UD}}=2.27$ mas in the $K$ band), and that beyond this value, i.e. in the second visibility lobe and further, limb-darkening and possibly energy at high spatial frequencies make the uniform disc model a poor approximation of the real spatial intensity distribution on the stellar disc. Therefore, in order to work with larger baselines, one has either to refine the reference source model or to go for smaller and hence fainter objects.

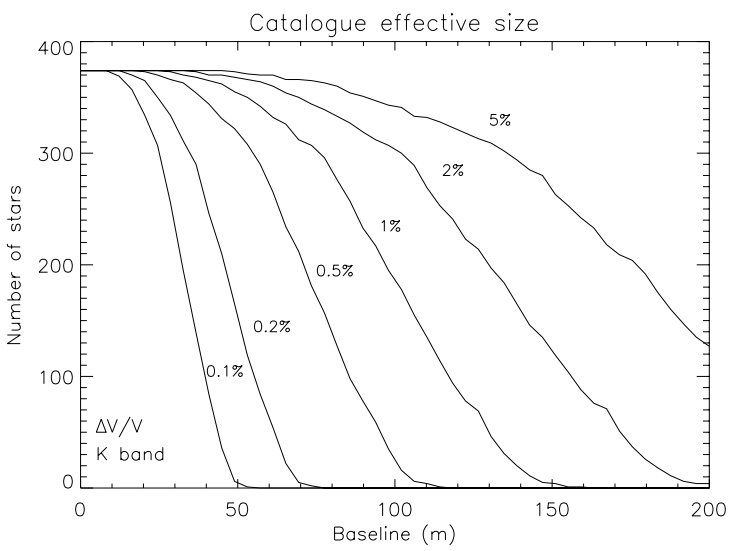

Fig. 9. Number of stars whose formal error on the UD diameter in the $K$ band is such that they are suitable for the required $\Delta V / V$ labeled on each curve.

The same issue can be tackled from a different viewpoint that links the required precision on the visibility to an "effective size" of the catalogue. Figure 9 represents as a function of the baseline, the number of stars whose relative precision on the UD diameter in the $K$ band is such that they are suitable for the required $\Delta V / V$ labeled on each curve (Table 3 is a numerical equivalent). In order to ensure easy measurements, we have retained only the stars whose visibilities would be above $20 \%$. For instance, if one tolerates a $2 \%$ precision on $V$, then 305 stars in the catalogue can be used as calibrators on a $100 \mathrm{~m}$ baseline. However, only 24 stars remain if a precision of $0.5 \%$ is needed.

Table 3. Number of stars whose formal error on the UD diameter in the $K$ band is such that they are suitable for the quoted $\Delta V / V$.

\begin{tabular}{ccccc}
\hline \hline & \multicolumn{4}{c}{$\Delta V / V$} \\
Baseline $(\mathrm{m})$ & $0.5 \%$ & $1 \%$ & $2 \%$ & $5 \%$ \\
\hline 50 & 316 & 354 & 366 & 372 \\
100 & 24 & 186 & 305 & 341 \\
150 & 0 & 4 & 126 & 266 \\
200 & 0 & 0 & 4 & 127 \\
\hline
\end{tabular}

\section{Interferometric catalogue}

\subsection{General presentation}

The catalogue is meant to group together all useful information about calibrator stars in a handy set. We have completed the original fields in C.X: HD number, spectral type, angular diameters and uncertainties on diameters with the equatorial coordinates, HR numbers, common names, parallaxes, proper motions, effective temperatures, surface gravities and Johnson's photometry $B, V$ and from $J$ to $N$. Two additional quantities useful in the FLUOR reduction process and defined in Sect. 5.3, namely the effective wavenumber and the shape factor, were computed in the case of an ideal interferometer and are provided for the $K^{\prime}$ band. Measurements have been compiled from different sources or estimated when missing (see Table 5 for 
details and references). Stars in the catalogue are ordered according to their HD number which we use as an identification number. The reasons why it is essential to keep track of the calibrator stars are twofold:

- to be able to track correlations between observations at different dates,

- if the model of the calibrator were to be modified for any reason, one would perhaps like to recompute the visibilities of some scientific targets, even years after the observations.

In this last section, we report how uniform disc angular diameters were computed, we define the effective wavenumber and the shape factor, and we describe the catalogue format.

\subsection{Angular diameters}

In their work, C.X derive stellar angular diameters with formal uncertainties by fitting Kurucz atmosphere models to composite spectra. As a consequence, such a diameter represents the true physical diameter of the star in the sense of the StefanBoltzmann law. It is not the uniform disc (UD) diameters that interferometrists are used to, but the UD diameter can be deduced from this true or limb-darkened (LD) diameter for a given spectral band provided the limb-darkening coefficients are known. We have computed UD diameters for $J, H$, and $K$ bands from the linear limb-darkening coefficients published by Claret et al. (1995) and the formula

$\frac{\theta_{\mathrm{LD}}}{\theta_{\mathrm{UD}}}=\sqrt{\frac{1-u_{\lambda} / 3}{1-7 u_{\lambda} / 15}}$.

introduced by Hanbury Brown (1974). Utilizing the spectral type and the luminosity class, the effective temperature $T_{\text {eff }}$ and surface gravity $\log g\left(g\right.$ in $\left.\mathrm{cm} \mathrm{s}^{-2}\right)$ are interpolated into the tables published respectively by de Jager \& Nieuwenhuijzen (1987) and Straizys \& Kuriliene (1981). In turn, the linear limb-darkening coefficient $u_{\lambda}$ is interpolated into Claret's tables using $T_{\text {eff }}$ and $\log g$ (see schema in Fig. 10 for a summary of the method). Assuming that the errors on the effective temperatures and surface gravities are respectively $\Delta T_{\text {eff }}=200 \mathrm{~K}$ (after comparison between various authors using different techniques: de Jager \& Nieuwenhuijzen 1987; Perrin et al. 1998 and van Belle et al. 1999) and $\Delta \log g=0.4$ (Straizys \& Kuriliene 1981), we find that the resulting error on the UD diameters is of the same order as the maximum error coming from Hanbury Brown's formula, i.e. $0.2 \%$. Consequently, the conversion process from LD diameters to UD diameters deteriorates only slightly their accuracy.

\subsection{Effective wavenumber and shape factor}

Equation (2) defines a monochromatic visibility depending on wavelength for two reasons: $\lambda$ appears obviously in (2), and also the star's diameter may be a function of $\lambda$ as different materials are observed at different wavelengths. As a consequence, it is necessary to define an effective wavelength or wavenumber for large bands observations (Perrin 1996). Interferometrists usually prefer to work with the wavenumber $\sigma$ since it is the
Fourier-conjugate variable of the optical path difference. If we admit that the squared visibility varies slowly with $\sigma$ in the spectral band of interest, then a first order Taylor expansion yields

$V^{2}(\sigma)=V^{2}\left(\sigma_{0}\right)+A\left(\sigma-\sigma_{0}\right)$,

where $A$ is a constant. If $B$ denotes the spectrum of the source multiplied by the filter transmission profile, then the measured large band visibility would be for a perfect interferometer

$$
\begin{aligned}
V_{\text {meas }}^{2} & =\frac{\int_{0}^{\infty} B^{2}(\sigma) V^{2}(\sigma) \mathrm{d} \sigma}{\int_{0}^{\infty} B^{2}(\sigma) \mathrm{d} \sigma} \\
& =V^{2}\left(\sigma_{0}\right)+A \frac{\int_{0}^{\infty}\left(\sigma-\sigma_{0}\right) B^{2}(\sigma) \mathrm{d} \sigma}{\int_{0}^{\infty} B^{2}(\sigma) \mathrm{d} \sigma}
\end{aligned}
$$

The effective wavenumber is chosen to cancel the first order term, so that the corresponding monochromatic visibility would be equal to the measured large band one (adapted from Golay 1974). Hence,

$\sigma_{\text {eff }} \equiv \frac{\int_{0}^{\infty} \sigma B^{2}(\sigma) \mathrm{d} \sigma}{\int_{0}^{\infty} B^{2}(\sigma) \mathrm{d} \sigma}$

As explained by Coudé du Foresto et al. (1997) who describe the FLUOR reduction process in details, the wide band squared fringe contrast measured by the instrument is weighted by the squared spectrum of the source:

$\overline{\mu^{2}}=\frac{\int_{0}^{\infty} \mu^{2}(\sigma) B^{2}(\sigma) \mathrm{d} \sigma}{\int_{0}^{\infty} B^{2}(\sigma) \mathrm{d} \sigma}$

If the reference and the target have identical spectra (are of the same spectral type), the denominator cancels out in Eq. (1) and $V_{\star}$ can be obtained without it. Otherwise, one has to go through its computation. As it embodies the differences in shape between the target and reference spectra, it is called the shape factor $(S F)$ :

$S F \equiv \int_{0}^{\infty} B^{2}(\sigma) \mathrm{d} \sigma$

The shape factor is then a cross property of the star and the instrument. We have computed the effective wavenumbers and shape factors in the $K^{\prime}$ band for all the stars in Cohen's list, thanks to the template files provided by the author (C.X). Let us point out the calculations were performed in the case of an ideal interferometer that introduces no chromatic effects (the instrumental transmission profile is spectrally flat). Under this approximation, $\sigma_{\text {eff }}$ and $S F$ depend only on the spectrum of the star multiplied by the profile of the filter (Fig. 11 shows the measured transmission profile of the $K^{\prime}$ filter used), and should be mostly considered as a relative information between stars of different spectral types. Both quantities does not vary much with the spectral type. Indeed, the effective wavenumber is contained between 4681 and $4687 \mathrm{~cm}^{-1}(\approx 0.1 \%$ variation with $\mathrm{Sp}$ ), whereas the shape factor (see Table 4) is contained between 13.14 and $13.22 \mu \mathrm{m}$, and vary less than $1 \%$ with $\mathrm{Sp}$. 


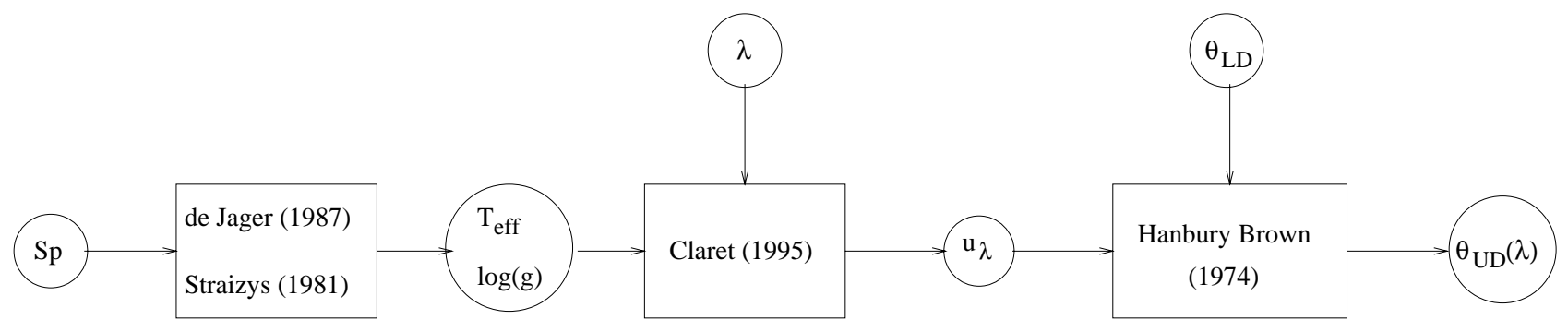

Fig. 10. Uniform disc (UD) diameter computation schema.

Table 4. Shape factor values corresponding to all spectral types appearing in our catalogue.

\begin{tabular}{ll}
\hline \hline $\mathrm{Sp}$ & $S F(\mu \mathrm{m})$ \\
\hline $\mathrm{K} 0-\mathrm{K} 0.5$ & 13.14 \\
$\mathrm{~K} 1-\mathrm{K} 2$ & 13.19 \\
$\mathrm{~K} 2.5-\mathrm{K} 3.5$ & 13.15 \\
$\mathrm{~K} 4.5-\mathrm{K} 8$ & 13.21 \\
$\mathrm{M} 0-\mathrm{M} 1$ & 13.22 \\
\hline
\end{tabular}

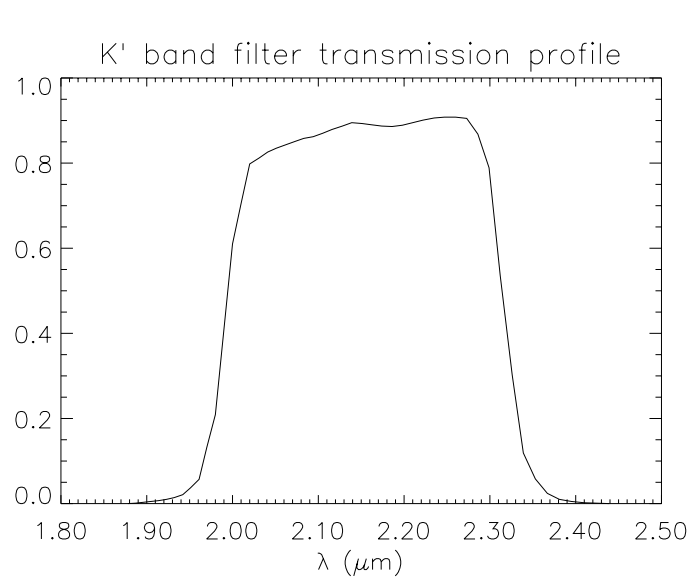

Fig. 11. $K^{\prime}$ band filter transmission profile.

\section{Comparison with other diameter estimates or measurements}

We have used the CHARM catalogue (Richichi \& Percheron 2002) to quickly search the literature for existing diameter estimates or measurements concerning stars in our selection. Different methods or instruments must be analyzed separately in order to reveal systematic effects at a precision of a few percent. We have chosen references for which the number of stars is high enough to allow some statistical analysis and the relative precision is below $10 \%$ (Table 6). As a result, some limb-darkened diameters $\theta_{\mathrm{LD}}$ can be compared with photometric estimates given by the infrared flux method: two sets of respectively 24 stars (Bell \& Gustafsson 1989) and 10 stars (Blackwell \& Lynas-Gray 1994) with 5 stars in common, and direct measurements performed by two interferometers: the Mark III (9 stars) and the NPOI (21 stars). Some UD diameters in the $K$ band, denoted $\theta_{\mathrm{UDK}}$, can be compared as well with direct measurements performed by two other interferometers: the IOTA ( 7 stars) and the PTI (10 stars). The six data sets will be investigated in this section.
Let us follow the analysis of Nordgren et al. (2001) for every data set. In order to look for biases, the mean $\overline{\Delta \theta}$ and its uncertainty $\sigma / \sqrt{N}$ are computed ( $\sigma$ denotes the standard deviation of the distribution $\Delta \theta$ and $N$ is the number of stars). Then, the reduced chi-square $\chi_{N}^{2}=\sum_{i=1}^{N}\left(\Delta \theta_{i} / \sigma_{i}\right)^{2} / N$ is evaluated in order to check the consistency of error bars. Finally, a linear least-square fit to the data is performed. If $\theta=a \times \theta_{\text {Cohen }}+b$, a perfect match would be $a=1$ and $b=0$ (Table 7, Figs. 12-14). On the whole, diameters computed by C.X and photometric estimates using the IRFM appear to be fully consistent. Since $\chi_{N}^{2}$ is found to be below one, we conclude like Nordgren et al. (2001) that the $4 \%$ precision claimed on the diameters for this method is indeed conservative.

The LD diameters measured by interferometry are also in very good agreement with the values of C.X. Although above one, the $\chi_{N}^{2}$ are still very low. Considering the small number of measures, it is difficult to draw a definitive conclusion about the compatibility of the error bars. However, the reader may find it appropriate to increase them by a factor $\sqrt{\chi_{N}^{2}}$. Besides, one may see a decreasing trend in Fig. 13b, that if confirmed will have to be explained.

Concerning the UD diameters in the $K$ band, the agreement is still good, but there are so few measures that the comparison is merely indicative. As a conclusion, our analysis show that there is no systematic bias between every pair of data sets, and that the error bars are reasonably compatible.

\section{Conclusion}

We have presented a catalogue of 374 stars carefully selected to be used as calibrator stars for long baseline stellar interferometry in the near infrared. This catalogue, a subset of the all-sky network of C.X established in the purpose of spectrophotometric calibration, takes advantage of its complete list of angular diameter with formal uncertainties and excellent sky coverage. The comparison between the angular diameters supplied in this catalogue and the measures published by various authors show an excellent agreement. Our catalogue provides additional information on the identification and position of the stars, as well as the Johnson photometry and other useful parameters. Depending on the required precision on the visibility and the wavelength, it is well suited for interferometers with maximum baselines between 100 and $200 \mathrm{~m}$.

Although great care has been put in the double selection process of these stars, first by C.X and then by us, the observational verification of this catalogue has hardly begun. 
Table 5. Description of catalogue fields. References are: $A Q=$ Astrophysical Quantities 4th ed, C95 = Claret et al. $(1995)$, C.X $=$ Cohen et al. (1999), HB74 = Hanbury-Brown et al. (1974), HIP = Hipparcos Catalogue (Perryman et al. 1997), J66 = Johnson (1966), JN87 = de Jager \& Nieuwenhuijzen (1987), Simbad (CDS), SK81 = Straizys \& Kuriliene (1981), VDS = Catalogue of visual double stars observed by Hipparcos (Dommanget \& Nys 2000). If a photometry flag is set to one, then the corresponding magnitude results from a measurement. On the contrary, if it is set to zero, the corresponding magnitude is an estimate based on the colors deduced from the spectral type SP (see AQ or J66).

\begin{tabular}{|c|c|c|c|c|}
\hline & Code & Units & Reference(s) & Description \\
\hline 1 & HD & & C.X & Henry Draper catalogue number \\
\hline 2 & HR & & C.X & Bright Star catalogue number, -1 otherwise \\
\hline 3 & NAME & & C.X & Bayer and/or Flamsteed name, HR or HD otherwise \\
\hline 4 & RA & HH.MMSSmmm & HIP & Right Ascension for Epoch 2000.0 \\
\hline 5 & DEC & sDD.PPSSmmm & HIP & Declination for Epoch 2000.0 \\
\hline 6 & PMRA & mas $\mathrm{yr}^{-1}$ & HIP & Proper motion along RA \\
\hline 7 & PMDEC & mas $\mathrm{yr}^{-1}$ & HIP & Proper motion along DEC \\
\hline 8 & PAR & mas & HIP & Parallax \\
\hline 9 & PARERR & mas & HIP & Uncertainty on PAR \\
\hline 10 & $\mathrm{SP}$ & & C.X & Spectral type and luminosity class \\
\hline 11 & LDD & mas & C.X & Limb-darkened disc diameter \\
\hline 12 & LDDERR & mas & C.X & Uncertainty on LDD \\
\hline 13 & TEFF & $\mathrm{K}$ & JN87 & Effective temperature \\
\hline 14 & GRAV & dimensionless & SK81 & Surface gravity $\log (g)\left(g\right.$ in $\left.\mathrm{cm} \mathrm{s}^{-2}\right)$ \\
\hline 15 & LDCJ & dimensionless & C95 & Linear limb-darkening coefficient for the $J$ band \\
\hline 16 & UDDJ & mas & HB74 & Uniform disc diameter in the $J$ band \\
\hline 17 & UDDJERR & mas & HB74 & Uncertainty on UDDJ \\
\hline 18 & $\mathrm{LDCH}$ & dimensionless & C95 & Linear limb-darkening coefficient for the $H$ band \\
\hline 19 & UDDH & mas & HB74 & Uniform disc diameter in the $H$ band \\
\hline 20 & UDDHERR & mas & HB74 & Uncertainty on UDDH \\
\hline 21 & LDCK & dimensionless & C95 & Linear limb-darkening coefficient for the $K$ band \\
\hline 22 & UDDK & mas & HB74 & Uniform disc diameter in the $K$ band \\
\hline 23 & UDDKERR & mas & HB74 & Uncertainty on UDDK \\
\hline 24 & EFFWNK & $\mathrm{cm}^{-1}$ & C.X & Effective wavenumber for the $K^{\prime}$ band \\
\hline 25 & SFK & $\mu \mathrm{m}$ & C.X & Shape factor for the $K^{\prime}$ band \\
\hline 26 & $\mathrm{~B}$ & mag & Simbad & Johnson's $B$ magnitude \\
\hline 27 & $\mathrm{~V}$ & mag & Simbad & Johnson's $V$ magnitude \\
\hline 28 & $\mathrm{~J}$ & mag & C.X or AQ & Johnson's $J$ magnitude \\
\hline 29 & FJ & boolean & & Flag on $J$ value \\
\hline 30 & $\mathrm{H}$ & mag & C.X or AQ & Johnson's $H$ magnitude \\
\hline 31 & $\mathrm{FH}$ & boolean & & Flag on $H$ value \\
\hline 32 & $\mathrm{~K}$ & mag & C.X or AQ & Johnson's $K$ magnitude \\
\hline 33 & FK & boolean & & Flag on $K$ value \\
\hline 34 & $\mathrm{~L}$ & mag & C.X or AQ & Johnson's $L$ magnitude \\
\hline 35 & FL & boolean & & Flag on $L$ value \\
\hline 36 & M & mag & C.X or AQ & Johnson's $M$ magnitude \\
\hline 37 & FM & boolean & & Flag on $M$ value \\
\hline 38 & $\mathrm{~N}$ & mag & J66 & Johnson's $N$ magnitude \\
\hline 39 & FN & boolean & & Flag on $N$ value \\
\hline 40 & COMTS & & Simbad, VDS & $\begin{array}{l}\text { Comments on the nature of the source, separations } \\
\text { and differences in magnitude for multiple stars }\end{array}$ \\
\hline
\end{tabular}

Table 6. References used for angular diameter comparisons. Symbols are $\theta_{\mathrm{LD}}$ for the limb-darkened diameter and $\theta_{\mathrm{UDK}}$ for the UD diameter in the $K$ band. IRFM = infrared flux method, LBSI = long baseline stellar interferometry, BG89 = Bell \& Gustafsson (1989), BL94 = Blackwell \& Lynas-Gray (1994), NS01 = Nordgren et al. (2001), NG99 = Nordgren et al. (1999), DV98 = Dyck et al. (1998), VB99 = van Belle et al. (1999).

\begin{tabular}{cccccc}
\hline \hline Method & Instrument & $\begin{array}{c}\text { Compared } \\
\text { diameter }\end{array}$ & $\begin{array}{c}\text { Average } \\
\text { precision }\end{array}$ & $\begin{array}{c}\text { Number } \\
\text { of stars }\end{array}$ & Reference(s) \\
\hline IRFM & - & $\theta_{\mathrm{LD}}$ & $4.0 \%$ & 24 & BG89 \\
IRFM & - & $\theta_{\mathrm{LD}}$ & $4.0 \%$ & 10 & BL94 \\
LBSI & Mark III & $\theta_{\mathrm{LD}}$ & $1.6 \%$ & 9 & NS01 \\
LBSI & NPOI & $\theta_{\mathrm{LD}}$ & $1.9 \%$ & 21 & NG99, NS01 \\
LBSI & IOTA & $\theta_{\mathrm{UDK}}$ & $8.4 \%$ & 7 & DV98 \\
LBSI & PTI & $\theta_{\mathrm{UDK}}$ & $2.8 \%$ & 10 & VB99 \\
\hline
\end{tabular}


Table 7. Result of the angular diameter comparisons: $N$ is the number of stars, $\overline{\Delta \theta}$ is the mean difference between the values given by C.X and the other determinations, $\sigma$ is the standard deviation of the distribution $\Delta \theta, \chi_{N}^{2}$ is the reduced chi-square, $a$ and $b$ are the coefficients of a linear least-square fit to the data $a \times \theta_{\text {Cohen }}+b$.

\begin{tabular}{ccccccc}
\hline \hline Determination & IRFM (BG89) & IRFM (BL94) & Mark III & NPOI & IOTA & PTI \\
\hline$N$ & 24 & 10 & 9 & 21 & 7 & 10 \\
$\overline{\Delta \theta}$ (mas) & $0.01 \pm 0.02$ & $0.04 \pm 0.02$ & $-0.06 \pm 0.05$ & $0.01 \pm 0.03$ & $0.21 \pm 0.24$ & $-0.08 \pm 0.05$ \\
$\sigma$ (mas) & 0.10 & 0.05 & 0.16 & 0.13 & 0.64 & 0.17 \\
$\chi_{N}^{2}$ & 0.5 & 0.4 & 2.4 & 3.0 & 1.4 & 6.7 \\
$a$ & $0.99 \pm 0.03$ & $0.97 \pm 0.05$ & $1.02 \pm 0.02$ & $1.04 \pm 0.01$ & $0.82 \pm 0.12$ & $0.81 \pm 0.06$ \\
$b$ & $0.05 \pm 0.09$ & $0.05 \pm 0.13$ & $-0.04 \pm 0.09$ & $-0.15 \pm 0.05$ & $1.05 \pm 0.84$ & $0.55 \pm 0.14$ \\
\hline
\end{tabular}
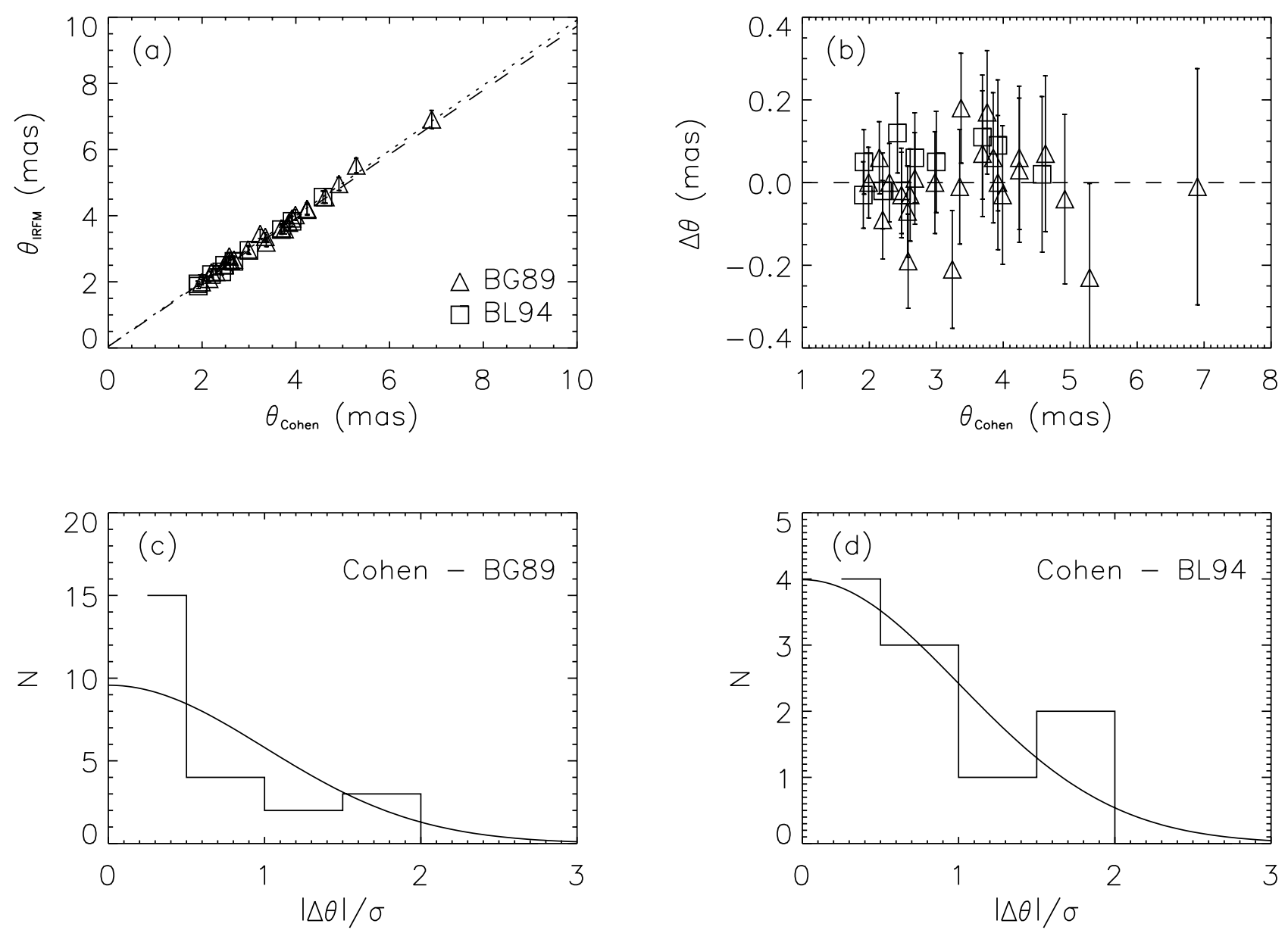

Fig. 12. Comparison with the IRFM: a) angulars diameters determined with the IRFM vs. values of C.X, b) their differences vs. values of C.X, c)-d) histograms of the folded distributions $|\Delta \theta| / \sigma$. Linear least-square fits and unit width Gaussians have been respectively overlaid for a) and c)-d). Two references have been used: BG89 = Bell \& Gustafsson (1989) and BL94 = Blackwell \& Lynas-Gray (1994).

In particular, undetected close companions may be discovered when a high level of a precision is attained. Moreover, as baselines larger than $200 \mathrm{~m}$ become available, the number of resolved sources for which the uniform disc model might not be accurate enough will increase significantly (see Fig. 9). More work is needed to extend this catalogue with sources suitable for longer baselines, such as the recently commissionned 330 m-baseline of the CHARA array (e.g. McAlister et al. 2000) or in a few years, the $800 \mathrm{~m}-$ maximum baseline of the 'OHANA project (e.g. Perrin et al. 2000). At the same time, this work would benefit to high accuracy instruments coming soon in line. In particular, the VLTI operated with AMBER (Petrov et al. 2000) is expected to attain a $0.1 \%$ relative visibility accuracy, necessary to carry out ambitious programs like the direct detection of extrasolar planets. 

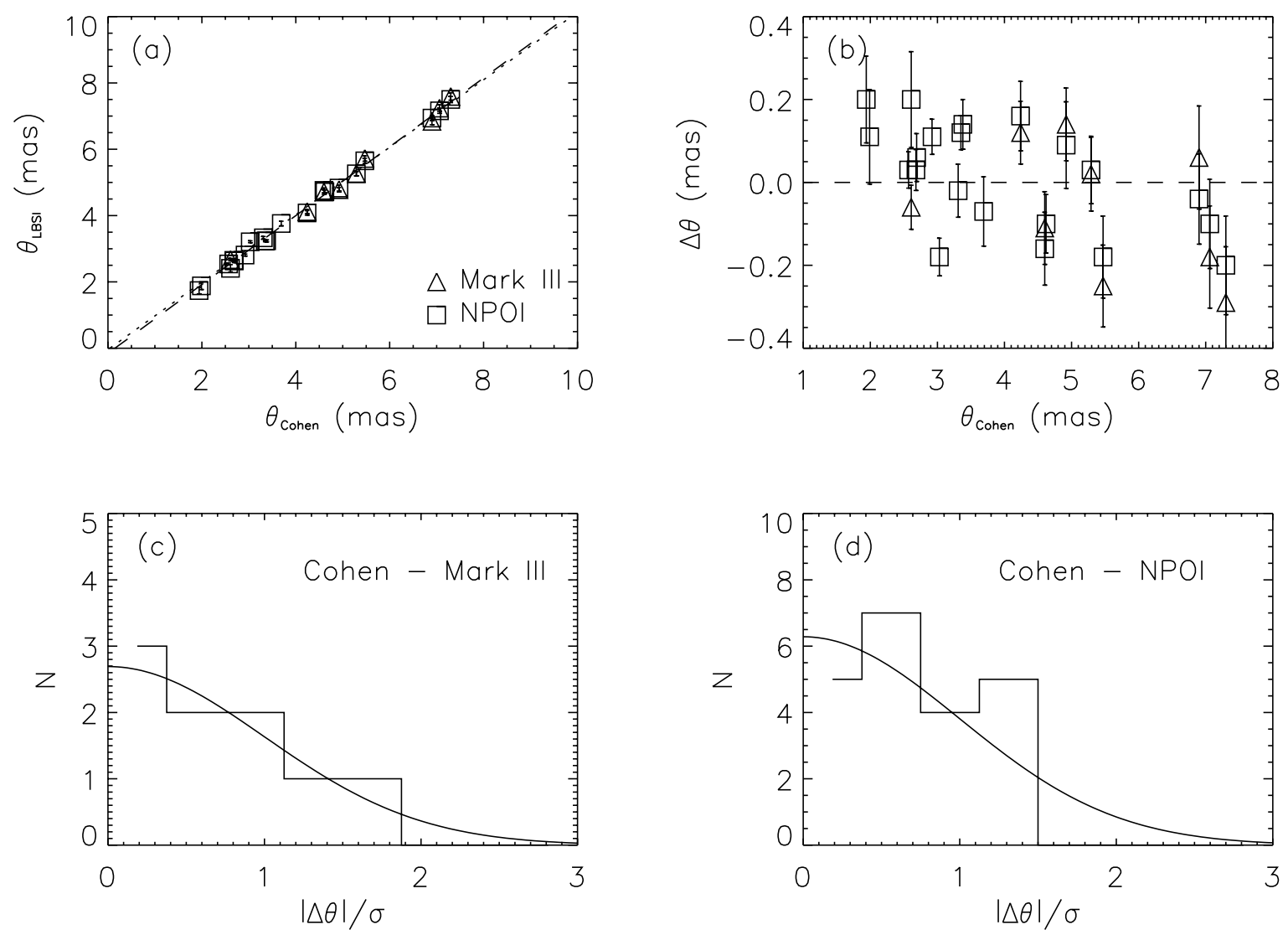

Fig. 13. Same as Fig. 12 with LD diameters measured by long baseline stellar interferometry.
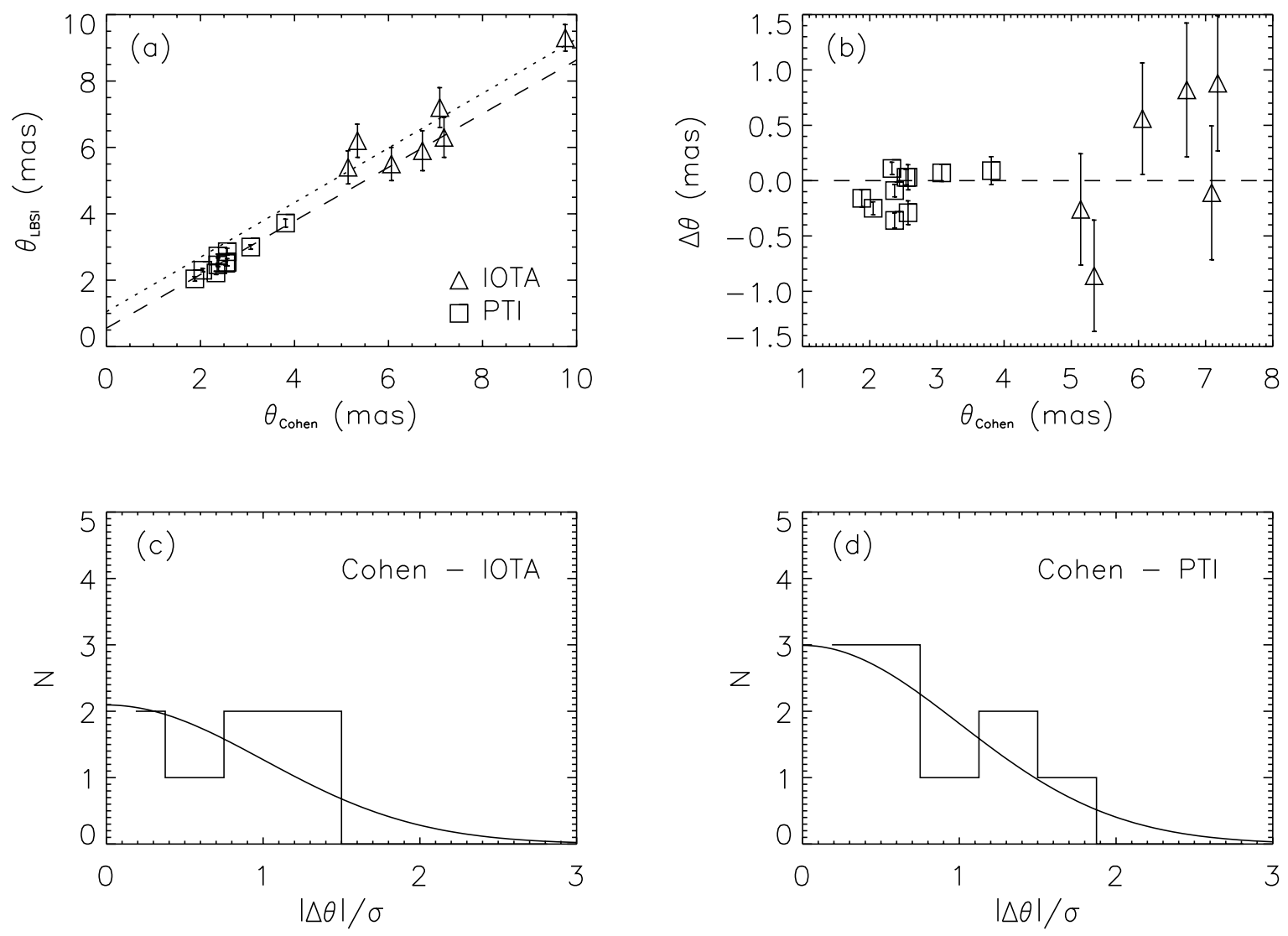

Fig. 14. Same as Fig. 12 with UD diameters in the $K$ band measured by long baseline stellar interferometry. 
Acknowledgements. The authors would like to thank Dr. Steve Ridgway for useful discussions and the referee, Dr. Andrea Richichi, for his constructive review of this paper. This research has made use of the Simbad database, operated at the Centre de Données Astronomiques de Strasbourg (CDS), and of NASA's Astrophysics Data System Bibliographic Services (ADS).

\section{References}

Allen's Astrophysical Quantities, Fourth Edition, ed. A. N. Cox (Springer-Verlag), 2000 (AQ)

Batten, A. H., Fletcher, J. M., \& MacCarthy, D. G. 1989, Catalogue of the orbital elements of spectroscopic binary systems: 8: 1989, Victoria: Dominion Astrophysical Observatory

Bell, R. A., \& Gustafsson, B. 1989, MNRAS, 236, 653 (BG89)

Blackwell, D. E., \& Lynas-Gray, A. E. 1994, A\&A, 282, 899 (BL94)

Boden, A., Creech-Eakman, M. J., \& Queloz, D. 2000, ApJ, 536, 880

Claret, A., Diaz-Cordoves, J., \& Gimenez, A. 1995, A\&AS, 114, 247 (C95)

Cohen, M., Witteborn, F. C., Carbon, D. F., et al. 1996, AJ, 112(5), 2274

Cohen, M., Walker, R. G., Carter, B., et al. 1999, AJ, 117, 1864 (C.X)

Colavita, M., Wallace, J. K., Hines, B. E., et al. 1999, ApJ, 510, 505

Dyck, H. M., van Belle, G. T., \& Thompson, R. R. 1998, AJ, 116, 981 (DV98)

Coudé Du Foresto, V., et al. 1997, A\&AS, 121, 379

Coudé Du Foresto, V., et al. 1998, SPIE, 3350, 856

Dommanget, J., \& Nys, O. 2000, A\&A, 363, 991 (VDS)

Engelke, C. W. 1992, AJ, 104 (3), 1248

Glindemann, A., et al. 2000, SPIE, 4006, 2

Golay, M. 1974, Introduction to Astronomical Photometry, vol. 41 in the Astrophysics and Space Science Library (Reidel, Dordrecht), 39
Hanbury Brown, R., Davis, J., Lake, R. J. W., \& Thompson, R. J. 1974, MNRAS, 167, 475 (HB74)

Hestroffer, D. 1997, A\&A, 327, 199

de Jager, C., \& Nieuwenhuijzen, H. 1987, A\&A, 177, 217 (JN87)

Johnson, H. L. 1966, ARA\&A, 4, 193 (J66)

Kurucz, R. L. 1993, CD-ROM series, ATLAS9 Stellar atmospher programs and $2 \mathrm{~km} \mathrm{~s}^{-1}$ grid (Kurucz CD-ROM No. 13), SYNTHE Spectrum synthesis programs and line data (Kurucz CD-ROM No. 18)

McAlister, H. A., Bagnuolo, W. G., ten Beummelaar, T. A., et al. 2000, SPIE, 4006, 465

Nordgren, T. E., Germain, M. E., Benson, J. A., et al. 1999, AJ, 118, 3032 (NG99)

Nordgren, T. E., Sudol, J. J., \& Mozurkewich, D. 2001, AJ, 122, 2707 (NS01)

Ochsenbein, F., \& Dubois, P. 1992, Astronomy from Large Databases, II, 405

Perrin, G. 1996, Ph.D. Thesis, Université Paris 7

Perrin, G., Coudé du Foresto, V., Ridgway, S. T., et al. 1998, A\&A, 331,619

Perrin, G., Lai, O., Lena, P. J., \& Coudé du Foresto, V. 2000, SPIE, 4006, 708

Perryman, M. A. C., Lindegren, L., Kovalevsky, J., et al. 1997, A\&A, 323, L49 (HIP)

Petrov, R., Malbet, F., Richichi, A., \& Hofmann, K.-H. 1998, ESO Messenger, 92, 11

Richichi, A., \& Percheron, I. 2002, A\&A, 386, 492 (CHARM)

Straizys, V., \& Kuriliene, G. 1981, Ap\&SS, 80, 353 (SK81)

Traub, W. A., Carleton, N. P., Bregman, J. D., et al. 2000, SPIE, 4006, 715

van Belle, G. T., Lane, B. F., Thompson, R. R., et al. 1999, AJ, 117, 521 (VB99)

Walker, R. G., \& Cohen, M. 1988, AJ, 95, 1801 